\title{
BMJ Open Exploring contextual factors influencing the implementation of evidence-based care for hypertension in Rwanda: a cross-sectional study using the $\mathrm{COACH}$ questionnaire
} Ana A Baumann ${ }^{10},{ }^{1}$ Cole Hooley, ${ }^{2}$ Charles W Goss, ${ }^{3}$ Vincent Mutabazi, ${ }^{4}$
Angela L Brown, ${ }^{5}$ Kenneth B Schechtman, ${ }^{6}$ Marc Twagirumukiza, ${ }^{4,7}$
Lisa de las Fuentes, ${ }^{8,9}$ Dominic Reeds,,${ }^{10}$ Makeda Williams, ${ }^{11}$ Eugene Mutimura,,${ }^{4,12}$
Anna Bergström, ${ }^{13}$ Aurore Nishimwe,,${ }^{4,7}$ Cecile Ingabire,,${ }^{4,7}$ Victor G Davila-Roman ${ }^{5}$

To cite: Baumann AA, Hooley C, Goss CW, et al. Exploring contextual factors influencing the implementation of evidencebased care for hypertension in Rwanda: a cross-sectional study using the $\mathrm{COACH}$ questionnaire. BMJ Open 2021;11:e048425. doi:10.1136/ bmjopen-2020-048425

- Prepublication history for this paper is available online. To view these files, please visit the journal online (http://dx.doi org/10.1136/bmjopen-2020048425).

Received 01 January 2021 Accepted 01 July 2021

Check for updates

(C) Author(s) (or their employer(s)) 2021. Re-use permitted under CC BY-NC. No commercial re-use. See rights and permissions. Published by BMJ.

For numbered affiliations see end of article.

Correspondence to Dr Victor G Davila-Roman; vdavila@wustl.edu

\section{ABSTRACT}

Importance Hypertension is the largest contributor to the Global Burden of Disease. In Rwanda, as in most low-income and middle-income countries, an increasing prevalence of hypertension and its associated morbidity and mortality is causing major healthcare and economic impact. Understanding healthcare systems context in hypertension care is necessary.

Objective To study the hypertension healthcare context as perceived by healthcare providers using the Context Assessment for Community Health (COACH) tool. Design A cross-sectional cohort responded to the $\mathrm{COACH}$ questionnaire and a survey about hypertension training. Setting Three tertiary care hospitals in Rwanda. Participants Healthcare professionals $(n=223)$. Primary outcome(s) and measure(s) The $\mathrm{COACH}$ tool consists of 49 items with eight subscales: resources, community engagement, commitment to work, informal payment, leadership, work culture, monitoring services for action (5-point Likert Scale) and sources of knowledge (on a $0-1$ scale). Four questions surveyed training on hypertension.

Results Responders ( $n=223,75 \%$ women; $56 \%$ aged 20-35 years) included nurses ( $n=142,64 \%$, midwives $(n=42,19 \%)$, primary care physicians $(n=28,13 \%)$ and physician specialists $(n=11,5 \%))$. The subscales commitment to work, leadership, work culture and informal payment scored between 4.7 and 4.1 and the community engagement, monitoring services for action and organizational resources scored between 3.1 and 3.5. Sources of knowledge had a mean score of $0.6 \pm 0.3$. While $73 \%$ reported having attended a didactic hypertension seminar in the past year, only $28 \%$ had received long-term training and $51 \%$ had $<3$-year experience working with hypertension care delivery. The majority (99\%) indicated a need for additional training in hypertension care. Conclusions There is a need for increased and continuous training in Rwanda. Healthcare responders stated a commitment to work and reported supportive leadership, while acknowledging limited resources and no monitoring systems. The COACH tool provides
Strengths and limitations of the study

- A cross-sectional cohort of providers from three hospitals in Rwanda responded to the Context Assessment for Community Health (COACH) tool and a survey examining existing training in hypertension care.

- Respondents shared a high commitment to work and positive leadership, while indicating the need for more training in hypertension care and monitoring.

- Based on the COACH tool training, strategies are being codeveloped to implement a comprehensive and sustainable hypertension care programme in Rwanda.

contextual guidance to develop training strategies prior to the implementation of a sustainable hypertension care programme.

\section{BACKGROUND}

Hypertension is the most common disease in the world affecting globally over 1.2 billion people, is the largest contributor to the Global Burden of Disease and is the most prevalent risk factor for the development of cardiovascular disease (CVD). ${ }^{1-3}$ The overall global prevalence of hypertension exceeds $50 \%$ of adults older than 50 years and in low-income and middle-income countries (LMICs) ranges from $32 \%$ to $78 \% .^{4-7}$ Rwanda, located in sub-Saharan Africa, is an LMIC with 12.4 million relatively young people (those $<34$ years of age represent $78 \%$ of the population) and with a high population density. ${ }^{78}$ Rwanda is undergoing an epidemiological transition with an increasing coexistence of infectious diseases and noncommunicable diseases (NCDs) including 
hypertension. ${ }^{89}$ Epidemiologic data from Rwanda estimates a high prevalence of both hypertension and CVD, which together account for $36 \%$ of deaths. ${ }^{10}$ The 2015 Rwanda NCDs risk survey based on the WHO STEPwise Approach to Surveillance framework reported an overall hypertension prevalence of $15 \%$ (95\% CI: 13.8 to 16.3 ) for those aged 15-64 years and 39\% (95\% CI: 35.7 to 43.1) for those aged 55-64 years, with deaths attributable to hypertension at 18/100 $000 .{ }^{11}$ These data are consistent with those of other sub-Saharan countries, reflecting the importance of hypertension as a public health burden in sub-Saharan Africa. ${ }^{12}$

While evidence-based intervention (EBI) studies have shown that treatment and control of hypertension decrease morbidity and mortality, barriers for the implementation of these interventions have been found at all healthcare levels, including systems, providers and patients. ${ }^{13}$ The application of dissemination and implementation (D\&I) science allows for a rigorous and systematic approach to develop implementation strategies and improve the uptake of effective EBIs for hypertension care. $^{14}$

Selection of implementation strategies, that is, methods to implement these EBIs in usual care, should be based on frameworks and on an understanding of the context where the intervention will be implemented. ${ }^{15}$ The Promoting Action on Research Implementation in Health Services (PARIHS) is a framework that outlines three core elements (evidence, context and facilitation) for successful EBI implementation. ${ }^{14} 15$ Context, defined as 'the environment or setting in which the proposed change is to be implemented', is comprised of three subelements: culture, leadership and evaluation, dimensions being evaluated in this study. ${ }^{14}$ The Context Assessment for Community Health $(\mathrm{COACH})$, a validated theory-based tool aimed at examining healthcare contexts in LMICs, is a 49-item survey based on the context dimension of the PARIHS framework and the interconnected building blocks of the WHO. ${ }^{16}$ The COACH tool was developed to identify barriers for EBI implementation, to guide planning and adaptation of the strategies to increase the uptake of the EBIs and to link contextual characteristics to outcome indicators of healthcare interventions. ${ }^{16}$ Due to its theoretical base and its acceptable reliability and validity among providers in a variety of LMICs including Vietnam, Bangladesh, Uganda, South Africa and Nicaragua, ${ }^{16} 17 \mathrm{COACH}$ was used in the present study to examine the context of hypertension care as reported by healthcare providers from three hospitals in Rwanda.

\section{METHODS}

\section{Study setting}

The study was conducted at three hospitals (two district, one provincial) in Rwanda; to maintain the confidentiality of participants, the hospitals are identified as A, B and $\mathrm{C}$.

\section{Participants}

Participants were healthcare providers working in the included hospitals. Lists of all medical professional staff (nursing, midwives, physicians) were provided by the hospital administrations to the study team; from these lists, study participants were contacted to participate in the study. All those who were contacted agreed to participate, signed informed consent and subsequently underwent in-person interviews to complete the survey.

\section{Questions about provider training in hypertension}

A separate survey asked healthcare providers four questions regarding training in hypertension care: (1) have you received didactic or school-based training on hypertension? (response options for first three questions: in the past year, before the past year, never); (2) have you received a structured long-term (>1-month course) training on hypertension?; (3) have you received on-job training, in service or supervision on the management of hypertension? and (4) do you feel there is need for additional training in the management of hypertension? (response options: yes, no/not sure).

\section{Questions about healthcare system context}

The COACH tool consists of 49 questions across eight subscales. Organizational resources refers to the availability of human capacity and materials that allow an organisation to implement an intervention successfully. Community engagement refers to mutual communication and activities that occur between community members and the organisation. Monitoring refers to the process of using locally derived data to evaluate performance and plans to improve outcomes. Sources of knowledge refers to the availability and use of sources of facts, information and skills acquired by providers through experience or education in an organisation that facilitate best practice. Commitment to work refers to an individual identification with and involvement in an organisation. Work culture refers to the process of an organisation, reflecting a shared set of values, ideas, concepts and rules of behaviour that allow the organisation to function. Leadership refers to the actions of a person in the organisation who can influence change and excellence in practice, achieved through clarity and engagement. Informal payment refers to bribe and/or benefits given to an individual outside of the officially accepted arrangements. ${ }^{16}$ Respondents were asked to rate their level of agreement using a 5-point Likert Scale for all items, except for items in the sources of knowledge subscale where the scale is from 0 to 1 , regarding the use of the hypertension guidelines within a specified time frame and the availability of different sources of knowledge.

\section{Translation and adaptation of COACH tool}

The majority of healthcare workers in Rwanda do not routinely communicate in English. For this reason, the COACH tool was translated from English to Kinyarwanda (study participants' daily language) and subsequently 
back translated to English by two Rwandan bilingual experts in both English and Kinyarwanda, following the WHO guidelines for translation of assessments ${ }^{18}$ to ensure the accuracy of the translation. Items that needed further clarification after pilot testing were discussed with the tool developer and agreement on the translation was reached by consensus. Additionally, we adapted the questions to be specific in relation to hypertension care (eg, 'This facility is willing to use new healthcare practices such as guidelines and recommendations for HTN').

\section{Patient and public involvement}

The COACH questionnaire has been previously validated in LMICs and was modified by the research team to address the context of hypertension in Rwanda through our weekly calls. The Rwandan coinvestigators had primary responsibility for the translation (English to Kinyarwanda) and back translation of the $\mathrm{COACH}$ questionnaire and for development, recruitment and conduction of the study. Patients were not involved in the development, recruitment or conduction of the study. The results of this study have been shared with stakeholders in Rwanda through our yearly meetings. Additional dissemination with the ministry of health, academic institutions, healthcare providers and others will occur once the manuscript is published.

\section{Data collection}

Data were collected by in-person interviews, using structured questionnaires in paper and pencil format, and subsequently transferred in duplicate entry to Qualtrics by members of the research team. There were no missing values for any of the questions and any discrepancies in data entry were resolved. The survey was administered between 27 May and 4 June 2019.

\section{Analysis}

Demographics are reported as the total number of respondents and percentage in each group. Differences among hospitals were assessed using $\chi^{2}$ tests or Fisher's exact tests as appropriate. $\mathrm{COACH}$ dimensions are reported as means $\pm \mathrm{SD}$, for each hospital and combined across all hospitals. Cronbach's $\alpha$, a coefficient of reliability, was used to determine internal consistency of the test items and the average intercorrelation between the items in each dimension. Scoring of sources of knowledge dimension ranged from 0 (not available, never/rarely), 0.5 (occasionally) to 1 (frequently/always); scoring for the other subscales ranged from 1 (strongly disagree) to 5 (strongly agree). The first six items in the informal payment dimension were reverse scored so that the directional interpretation was similar to the other dimensions.

A one-way analysis of variance was used to test for differences in mean dimension scores among the three hospitals. All analyses were conducted using SAS V.9.4 (SAS Institute) and $p$ values $<0.05$ were considered significant.

\section{RESULTS}

The total number of respondents $(n=223)$ included nurses, midwives, primary care physicians and specialty physicians, with approximately similar representation obtained from all three hospitals (table 1). The majority of respondents were female, relatively young, had nursing or midwife degrees and had limited experience working in hypertension care. The hospitals differed in terms of number of years of experience of the healthcare providers working in hypertension care $(\mathrm{p}=0.003)$.

\section{Training in hypertension}

Results of the perspectives of healthcare providers regarding training in hypertension care show that nearly all respondents (99\%) have participated in a didactic or school-based training on hypertension at some point during their training and/or career (either in the past year or before the past year, table 2). Almost half (44\%) of respondents stated having participated in long-term training (ie, $>1$-month course) on hypertension (either in the past year or before the past year) and a significant majority (72\%) had received on-job training, in service or supervision on the management of hypertension at some

Table 1 Participant demographics reported as $\mathrm{n}(\%)$

\begin{tabular}{lllllll}
\hline Variable & Group & $\begin{array}{l}\text { All } \\
(\mathbf{N}=223)\end{array}$ & $\begin{array}{l}\text { Hospital A } \\
(\mathbf{N}=74)\end{array}$ & $\begin{array}{l}\text { Hospital B } \\
(\mathbf{N = 7 0 )}\end{array}$ & $\begin{array}{l}\text { Hospital C } \\
\text { (N=79) }\end{array}$ & P value \\
\hline Age (years) & $20-35$ & $125(56 \%)$ & $40(54 \%)$ & $40(57 \%)$ & $45(57 \%)$ & 0.914 \\
& $\geq 36$ & $98(44 \%)$ & $34(46 \%)$ & $30(43 \%)$ & $34(43 \%)$ & \\
Gender & Female & $168(75 \%)$ & $51(69 \%)$ & $53(76 \%)$ & $64(81 \%)$ & 0.2 \\
Education & Nurse & $142(64 \%)$ & $46(62 \%)$ & $47(67 \%)$ & $49(62 \%)$ & 0.811 \\
& Midwife & $42(19 \%)$ & $17(23 \%)$ & $11(16 \%)$ & $14(18 \%)$ & \\
& Primary care physician & $28(13 \%)$ & $9(12 \%)$ & $9(13 \%)$ & $10(13 \%)$ & \\
Experience working in hypertension care & Physician specialist & $11(5 \%)$ & $2(3 \%)$ & $3(4 \%)$ & $6(8 \%)$ & \\
(years) & $0-3$ & $114(51 \%)$ & $32(43 \%)$ & $28(40 \%)$ & $54(68 \%)$ & 0.003 \\
& $4-9$ & $64(29 \%)$ & $27(37 \%)$ & $22(31 \%)$ & $15(19 \%)$ & \\
\hline
\end{tabular}


Table 2 Participant hypertension training history reported as number and per cent of those responding 'yes'

\begin{tabular}{|c|c|c|c|c|c|}
\hline Variable & All ( $\mathrm{N}=223)$ & Hospital A ( $\mathrm{N}=74)$ & Hospital B ( $\mathrm{N}=70)$ & Hospital C ( $=79)$ & $P$ value \\
\hline \multicolumn{6}{|c|}{ Have you received didactic or school-based training on hypertension? } \\
\hline a. In the past year & $72(32 \%)$ & $34(46 \%)$ & $24(34 \%)$ & $14(18 \%)$ & $<0.001$ \\
\hline c. Never & $3(1 \%)$ & $2(3 \%)$ & $1(1 \%)$ & 0 & 0.311 \\
\hline \multicolumn{6}{|c|}{ Have you received a structured long-term (>1-month course) training on hypertension? } \\
\hline b. Before the past year & $58(26 \%)$ & $28(38 \%)$ & $16(23 \%)$ & $14(18 \%)$ & 0.014 \\
\hline c. Never & $160(72 \%)$ & $45(61 \%)$ & $54(77 \%)$ & $61(77 \%)$ & 0.038 \\
\hline \multicolumn{6}{|c|}{ Have you received on-job training, in service or supervision on the management of hypertension? } \\
\hline a. In the past year & $81(36 \%)$ & $33(45 \%)$ & $30(43 \%)$ & $18(23 \%)$ & 0.008 \\
\hline b. Before the past year & $79(36 \%)$ & $31(42 \%)$ & $26(37 \%)$ & $22(28 \%)$ & 0.18 \\
\hline Yes & 220 (99\%) & 73 (99\%) & 70 (100\%) & 77 (97\%) & 0.776 \\
\hline
\end{tabular}

Note. Responses may vary in total numbers because participants were given the option to check all that apply.

time in their career (either in the past year or before the past year).

The level of training in hypertension care delivery varied by hospital, showing statistically significant differences in having received didactic or school-based training on hypertension in the past year and before the past year ( $<<0.001$ for both), in having received long-term training ( $>1$ month) before the past year $(\mathrm{p}=0.014)$ and having received on-job training, in service or supervision on the management of hypertension in the past year $(p=0.008)$ and never $(p=0.002)$. Finally, almost all respondents reported the need for additional training in hypertension $(99 \%)$ without statistically significant differences between hospitals.

\section{Internal reliability of COACH tool}

Overall, the COACH tool showed very good to high internal consistency (Cronbach's $\alpha$ range: 0.57-0.92; table 3) with all but three dimensions exceeding the accepted standard for satisfactory internal reliability of $>0.70$ for new scales. The highest Cronbach's $\alpha$ estimate (0.92) was for leadership subscale and the lowest (0.57) was for informal payment subscale. After removal of two items from the informal payments subscale (ie, 'Efforts are made to stop clients from providing informal payment to get appropriate healthcare services in hypertension' and 'Efforts are made to stop health workers from asking clients for informal payment for hypertension'), Cronbach's $\alpha$ increased to 0.74 . Similarly, for the work culture subscale, removal of one item (ie, 'This facility helps me to improve and develop my skills in hypertension') resulted in a Cronbach's $\alpha$ increase from 0.69 to 0.75 . Finally, for sources of knowledge subscale, removal of any of the constituent items did not improve Cronbach's $\alpha$ from 0.67 .

\section{COACH subscales}

Table 4 shows the mean and SDs for the scales across hospitals. There were significant differences in the mean scores across hospitals between the organizational

Table 3 Cronbach's $\alpha$ estimates for the different $\mathrm{COACH}$ dimensions

\begin{tabular}{|c|c|c|c|}
\hline Dimension & Items & $\begin{array}{l}\text { Score } \\
\text { range }\end{array}$ & $\begin{array}{l}\text { Cronbach's } \\
\alpha\end{array}$ \\
\hline $\begin{array}{l}\text { Organizational } \\
\text { resources }\end{array}$ & 11 & $1-5$ & 0.83 \\
\hline $\begin{array}{l}\text { Community } \\
\text { engagement }\end{array}$ & 5 & $1-5$ & 0.80 \\
\hline $\begin{array}{l}\text { Monitoring services for } \\
\text { action }\end{array}$ & 5 & $1-5$ & 0.74 \\
\hline Commitment to work & 3 & $1-5$ & 0.77 \\
\hline Work culture & 6 & $1-5$ & 0.69 \\
\hline $\begin{array}{l}\text { Work culture, with } \\
\text { questions removed }\end{array}$ & 5 & $1-5$ & 0.75 \\
\hline Leadership & 6 & $1-5$ & 0.92 \\
\hline Informal payment & 8 & $1-5$ & 0.57 \\
\hline $\begin{array}{l}\text { Informal payment, with } \\
\text { questions removedt }\end{array}$ & 6 & $1-5$ & 0.74 \\
\hline Sources of knowledge & 5 & $0-1$ & 0.67 \\
\hline
\end{tabular}

*Item: 'This facility helps me to improve and develop my skills in hypertension' removed.

†Items: 'Efforts are made to stop clients from providing informal payment to get appropriate healthcare services in hypertension' and 'Efforts are made to stop health workers from asking clients for informal payment for hypertension' were removed. $\mathrm{COACH}$, Context Assessment for Community Health. 
Table 4 Overall mean \pm SD and by hospital for the COACH subscales

\begin{tabular}{|c|c|c|c|c|c|}
\hline Dimensions & All $(\mathrm{N}=223)$ & Hospital A ( $N=74)$ & Hospital B ( $N=70)$ & Hospital C ( $=79)$ & $P$ value \\
\hline Organizational resources & $3.1 \pm 0.6$ & $3.3 \pm 0.8$ & $2.9 \pm 0.5$ & $3.1 \pm 0.5$ & $<0.001$ \\
\hline Human resources & $2.6 \pm 1.0$ & $3.2 \pm 1.1$ & $2.4 \pm 0.9$ & $2.3 \pm 0.8$ & $<0.001$ \\
\hline Communication and transport & $3.3 \pm 1.0$ & $3.3 \pm 1.1$ & $3.2 \pm 1.0$ & $3.4 \pm 0.8$ & 0.699 \\
\hline Financing & $2.9 \pm 0.7$ & $3.1 \pm 0.8$ & $2.7 \pm 0.6$ & $2.9 \pm 0.5$ & $<0.001$ \\
\hline Community engagement & $3.5 \pm 0.7$ & $3.5 \pm 0.8$ & $3.4 \pm 0.7$ & $3.6 \pm 0.5$ & 0.118 \\
\hline Monitoring services for action & $3.2 \pm 0.6$ & $3.3 \pm 0.8$ & $3.0 \pm 0.6$ & $3.2 \pm 0.5$ & 0.001 \\
\hline Commitment to work & $4.2 \pm 0.7$ & $4.3 \pm 0.6$ & $4.2 \pm 0.7$ & $4.1 \pm 0.8$ & 0.155 \\
\hline Work culture & $4.1 \pm 0.5$ & $4.0 \pm 0.7$ & $4.0 \pm 0.5$ & $4.3 \pm 0.4$ & 0.002 \\
\hline Culture of learning and change & $3.8 \pm 0.6$ & $3.8 \pm 0.7$ & $3.8 \pm 0.7$ & $3.7 \pm 0.5$ & 0.768 \\
\hline Accountability & $2.8 \pm 1.5$ & $2.7 \pm 1.5$ & $3.5 \pm 1.3$ & $2.3 \pm 1.3$ & $<0.001$ \\
\hline Informal payment* & $4.7 \pm 0.5$ & $4.6 \pm 0.6$ & $4.8 \pm 0.4$ & $4.8 \pm 0.3$ & 0.002 \\
\hline Nepotism* & $4.6 \pm 0.6$ & $4.5 \pm 0.7$ & $4.6 \pm 0.6$ & $4.7 \pm 0.6$ & 0.087 \\
\hline Sources of knowledge $†$ & $0.6 \pm 0.3$ & $0.6 \pm 0.3$ & $0.5 \pm 0.3$ & $0.7 \pm 0.3$ & 0.003 \\
\hline
\end{tabular}

All values represent mean \pm SD. Bolded items indicate $\mathrm{COACH}$ subscales; those below the subscales indicate the dimensions within the subscales.

*Items on informal payment and nepotism were reverse scored.

†Score range for sources of knowledge is $0-1$; for all other subscales the score range is 1-5.

$\mathrm{COACH}$, Context Assessment for Community Health.

resources, monitoring services, sources of knowledge, work culture, leadership and informal payment subscales.

The organizational resources received a mean score of 3.1, suggesting that respondents were overall neutral about this scale (tables 4 and 5 ). The majority of respondents agreed with only 3 of the 11 dimensions, including 'facility has access to transport and fuel that are needed to provide healthcare services for HTN' (50\% agreed), 'facility has access to the communication tools that are needed to provide healthcare services for HTN (54\%) and 'facility has enough disposable medical equipment to provide healthcare services for HTN' (83\% agreed). For the remaining eight dimensions, greater than 50\% of respondents were either neutral or disagreed with the dimensions dealing with human resources, space, medicine and equipment and financing, suggesting that there is room for improvement in these areas.

The community engagement received a mean score of 3.5, suggesting that respondents were slightly more favourable than neutral about the commitment of their hospitals towards their community (table 5). In fact, four of the five dimensions had more than half of the respondents agreeing with community engagement in hypertension care, with only one dimension, 'in this facility, we encourage other organizations to contribute to improving HTN in the community' showing a majority being either neutral $(36 \%)$ or disapproving $(27 \%)$.
The monitoring services for action received a mean score of 3.2, suggesting that respondents were neutral about the use of locally derived data to evaluate the performance and plans to improve hypertension care. Of the five dimensions, only one, 'this facility regularly compares its work with national or other guidelines for HTN' (61\%) showed agreement by more than half of the respondents.

The commitment to work received a mean score of 4.2, suggesting that respondents are committed to their hospitals. All three dimensions showed approval by a significant majority (ranging from $70 \%$ to $94 \%$ ).

The leadership subscale received a mean score of 4.1, suggesting that respondents are supportive of their leadership. All six dimensions had more than half of the respondents (81\%-92\%) agreeing with high remarks for their leadership.

The informal payment received a mean score of 4.7 , suggesting that respondents do not have concerns with informal payments, nepotism or accountability. In fact, in six of the eight dimensions, more than half of the respondents $(83 \%-98 \%)$ indicated no concerns with these issues. However, in the dimension of accountability, the low mean score of 2.8 is also reflected by a majority of combined neutral and disagree responses regarding 'efforts are made to stop clients from providing informal payment to get appropriate healthcare services in HTN' (22\% and 44\%, 


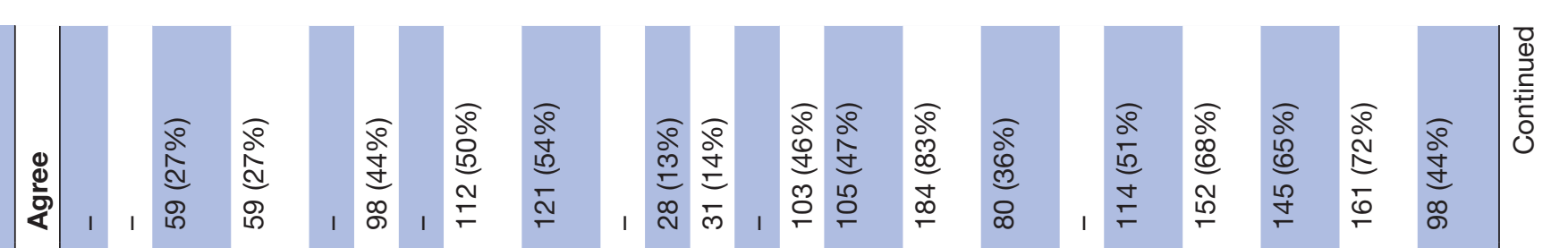

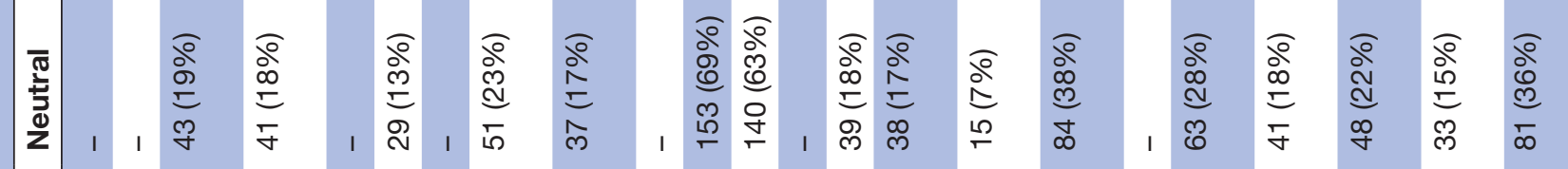

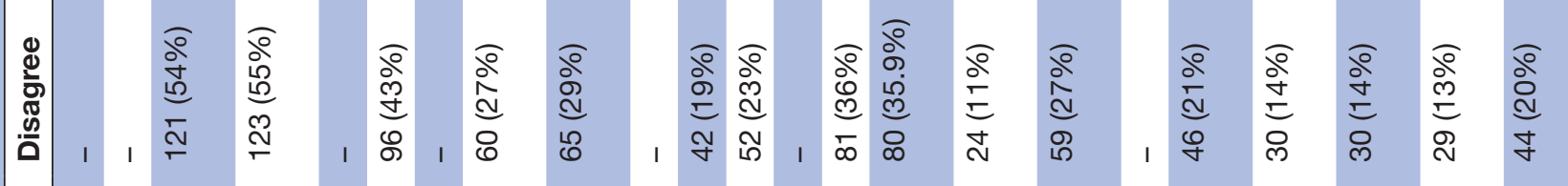

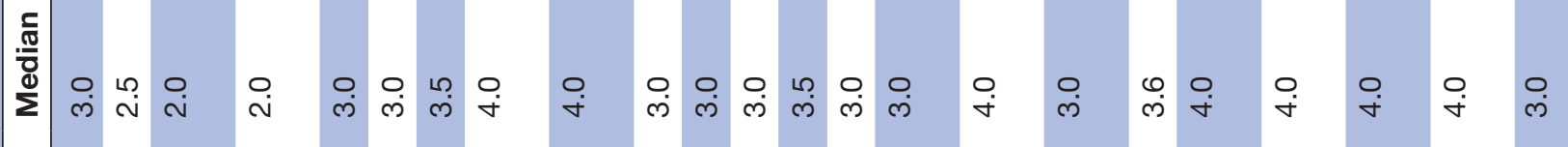

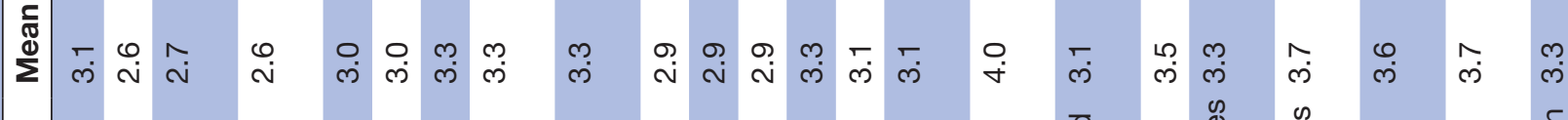




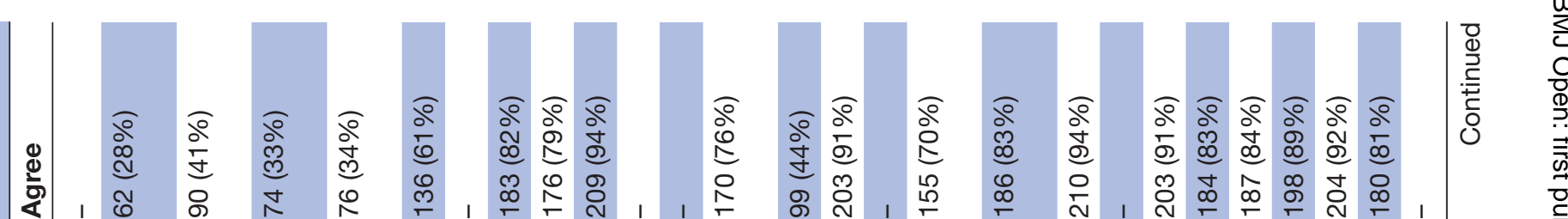

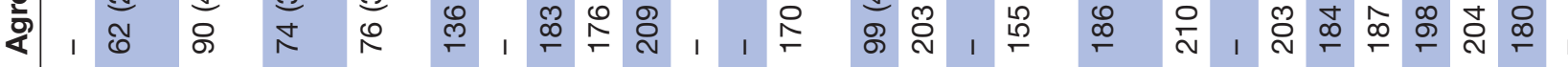

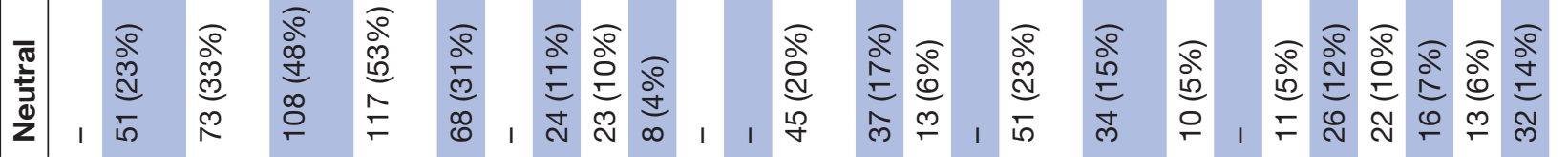

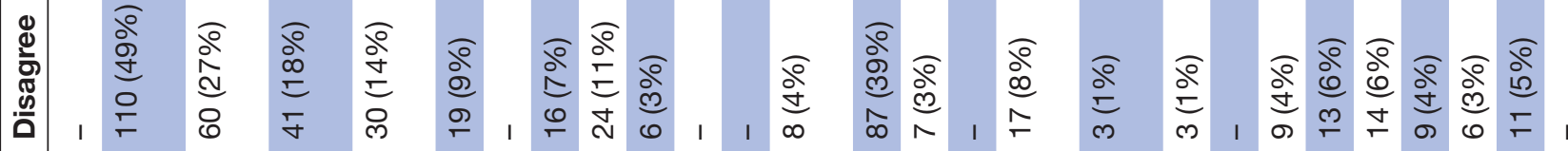

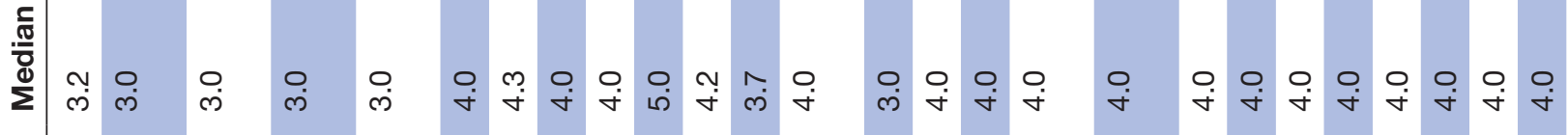

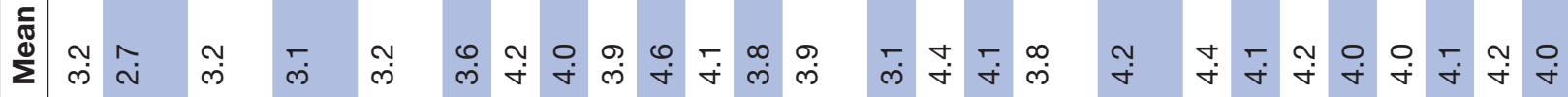

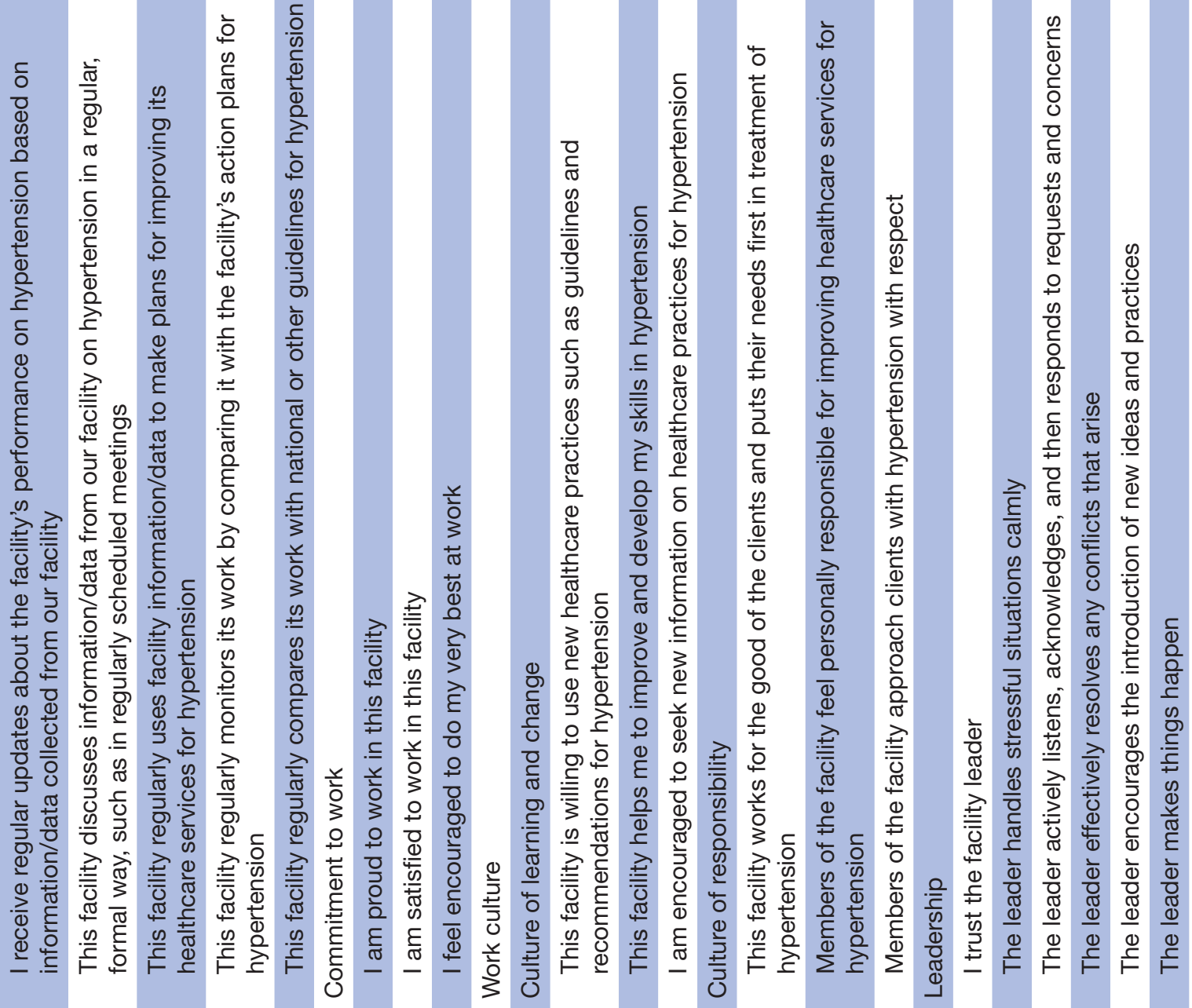




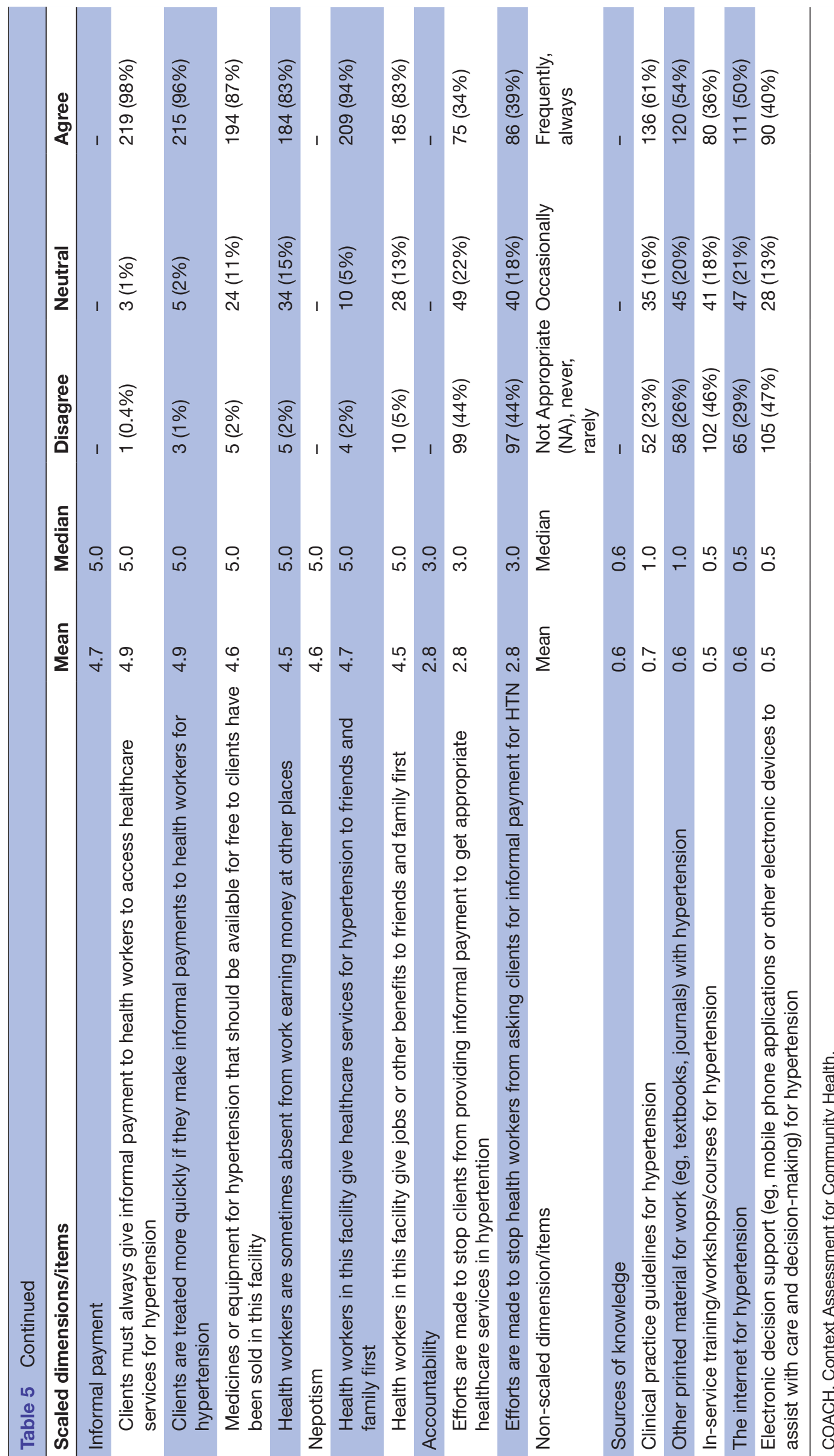


respectively) and 'efforts are made to stop health workers from asking clients for informal payment for HTN' (18\% and $44 \%$, respectively).

The sources of knowledge received a mean score of 0.6 (scale range: range $0-1$ ), with discordant result in three of the five dimensions: while a majority agreed that they have access to information regarding hypertension guidelines, stating agreement for 'clinical practice guidelines for HTN' $(61 \%)$, 'printed material for work with HTN' (54\%) and use of 'internet for HTN' (50\%), the majority responded with either occasionally or never/rarely responses regarding 'in-service training/workshops/courses for HTN' (18\% and $46 \%$, respectively) and 'electronic decision support for HTN' (13\% and 47\%, respectively).

\section{DISCUSSION}

The purpose of this study was to examine the contextual factors influencing hypertension care at three provincial/ district hospitals in Rwanda, ascertained after completion of the COACH tool, a survey validated for use in LMIC, and a questionnaire about training in hypertension. The main findings of the study indicate an overwhelming agreement from the majority of respondents for increased and/or continuous training in hypertension care, as ascertained in the training questionnaire and in the COACH knowledge and organizational resources subscales.

Our research team has been collaborating with healthcare providers from these hospitals for several years prior to survey, which may explain the high scores in the work culture and leadership subscales. While healthcare respondents in general stated a commitment to work and reported supportive leadership, the lower scores in the resources and monitoring services scales indicate challenges and opportunities for growth in these areas. In other words, even with such strong leadership in support of hypertension care, only about half of the respondents agreed with items that stated that the hospital had enough workers with the proper training and skills for HTN care. Additionally, the low scores on the monitoring subscale indicate that respondents believe that the hospital could improve evaluations of personnel performance with the purpose of improving hypertension outcomes.

These findings provide us with contextual guidance for the development of training strategies prior to the implementation of a comprehensive and sustainable hypertension care programme in Rwanda. That is, based on the data, the training programme should focus on providing further knowledge and understanding of the hypertension guidelines and on developing strategies to increase human capacity and monitoring of skills around hypertension care in these hospitals. The results indicate that we need to develop implementation strategies to support hypertension care in these hospitals and to think about how to increase skills on hypertension care and establish a monitoring system to support guideline adherence. The findings of this study reflect a larger historical context in Rwanda, with a shortage of trained physicians trained, especially after the 1996 genocide. ${ }^{19}$ Accordingly, recently there has been a national movement for nurse-led task sharing of HTN care. ${ }^{19} 20$

The findings of the COACH tool will guide our next steps in developing capacity building strategies and supporting the hospitals in establishing monitoring systems for HTN care. However, the informal payment and sources of knowledge subscales exhibited low Cronbach's $\alpha$ internal reliability scores. Other studies have found similar challenges with these subscales indicating that additional work is needed in these subscales. ${ }^{21}$ Regarding the subscale work culture, it is unclear why the item 'This facility helps me to improve and develop my skills in hypertension' was challenging in our study. Further cognitive interview with the Kinyarwanda translation of the tool is needed to understand challenges with this item.

There were significant differences in the mean scores across hospitals between the organizational resources, monitoring services, sources of knowledge, work culture, leadership and informal payment subscales, indicating the tool's ability to identify differences in these contextual factors. Assessment of the context of three hospitals provides guidance for our next training, selection and adaptation of implementation strategies to improve hypertension care in three hospitals in Rwanda. The overall good validity of the COACH tool indicates that it is comprehensible in Kinyarwanda and able to capture differences across settings.

The COACH tool was originally developed in five LMICs (Bangladesh, Vietnam, Uganda, South Africa, Nicaragua) and more recently applied in Mozambique to assess healthcare context and its potential use for integration of EBIs and to develop their implementation in clinical practice in LMICs. ${ }^{16}{ }^{17}$ The internal consistency of the COACH tool in a sample of providers in Rwanda, a different country from where the tool was developed and tested, show its utility to measure contextual dimensions in another LMIC. As this study is part of long-term capacity building efforts to strengthening D\&I science and hypertension science in Rwanda, our team is currently supporting the development of several studies, led by investigators in Rwanda, to examine implementation strategies to support the hypertension care in these hospitals.

\section{Limitations of the study}

While we gathered important information for our next steps in terms of context and the need for additional training in hypertension care, we did not examine the specific areas where the respondents need training in terms of hypertension care (eg, epidemiology, diagnosis, treatment). To further examine the hypertension care in these hospitals, we will gather qualitative data through focus groups with our stakeholders. Additionally, while between hospitals analyses of the scores were done, our team is unable to report these to avoid breach of confidentiality. This information has been shared with hospital leadership in a confidential manner so they can incorporate and support hypertension training in their strategic planning. Finally, the internal 
consistency of the COACH subscales after removal of the items was satisfactory, but further studies need to examine the challenges in the informal payment subscale and its low Cronbach's $\alpha$, also reported in other studies. ${ }^{17}$

\section{CONCLUSIONS}

Contextual measures related to hypertension care in Rwanda shows a need for increased and/or continuous training. Healthcare responders stated a general commitment to work and reported supportive leadership while acknowledging challenges with resources and monitoring services. The COACH tool provides contextual guidance for the development of training strategies prior to the implementation of a comprehensive and sustainable hypertension care programme in Rwanda.

\section{Author affiliations}

${ }^{1}$ Brown School of Social Work, Washington University in St. Louis, St. Louis, Missouri, USA

${ }^{2}$ School of Social Work, Brigham Young University, Provo, Utah, USA

${ }^{3}$ Division of Biostatistics, Washington University in St. Louis, St Louis, Missouri, USA

${ }^{4}$ Regional Alliance for Sustainable Development, Kigali, Rwanda

${ }^{5}$ Cardiovascular Division, Department of Medicine, Washington University in St.

Louis, St Louis, Missouri, USA

${ }^{6}$ Division of Biostatistics, Washington University in St. Louis, St. Louis, Missouri, USA

${ }^{7}$ College of Medicine and Health Sciences, University of Rwanda, Kigali, Rwanda

${ }^{8}$ Washington University in St. Louis, Division of Biostatistics, St. Louis, MO, USA

${ }^{9}$ Washington University in St. Louis, School of Medicine, Cardiovascular Division,

Department of Medicine, St. Louis, MO, USA

${ }^{10}$ Center for Human Nutrition, Division of Geriatrics and Nutritional Science,

Department of Medicine, St. Louis, M0, USA

${ }^{11}$ National Heart Lung and Blood Institute, Bethesda, Maryland, USA

${ }^{12}$ National Council for Science and Technology, Kigali, Rwanda and to Regional

Alliance for Sustainable Development, Kigali, Rwanda

${ }^{13}$ Uppsala Global Health Research on Implementation and Sustainability (UGHRIS),

Dept of Women's and Children's Health, Uppsala Universitet, Uppsala, Sweden

\section{Twitter Ana A Baumann @BaumannAna and Cole Hooley @cole_hooley}

Acknowledgements The authors acknowledge all providers who responded to the survey.

Contributors All authors conceptualised the study. ABaumann, $\mathrm{CH}$ and VM organised the survey. VM led Context Assessment for Community Health tool translation and back translation and was responsible for data collection. $\mathrm{CH}$ was responsible for data management. CWG was responsible for data analysis. ABaumann, CH, CWG and VGD-R drafted initial version of manuscript. All coauthors read, contributed to and approved the final manuscript.

Funding This work was supported in part by NHLBI grants U24HL136790, D43TW10335, 3U01HL133994 and UL1TR00234.

Disclaimer The views expressed in this manuscript are those of the authors and do not necessarily represent the views of the National Heart, Lung, and Blood Institute; the National Institutes of Health; or the U.S. Department of Health and Human Services.

\section{Competing interests None declared.}

Patient and public involvement Patients and/or the public were not involved in the design, or conduct, or reporting, or dissemination plans of this research.

\section{Patient consent for publication Not required.}

Ethics approval This study was approved by the University of Rwanda CMHS Institutional Review Board (approval notice: 099/CMSH IRB 2019) and Washington University School of Medicine Institutional Review Board Committee (IRB \# 201807048). Participants were informed of the potential risks and benefits of the study and signed an informed consent form in Kinyarwanda language before enrolment in the study. All participant data were anonymised to optimise privacy and confidentiality of responses.
Provenance and peer review Not commissioned; externally peer reviewed.

Data availability statement Data are available upon reasonable request to the corresponding author.

Open access This is an open access article distributed in accordance with the Creative Commons Attribution Non Commercial (CC BY-NC 4.0) license, which permits others to distribute, remix, adapt, build upon this work non-commercially, and license their derivative works on different terms, provided the original work is properly cited, appropriate credit is given, any changes made indicated, and the use is non-commercial. See: http://creativecommons.org/licenses/by-nc/4.0/.

ORCID iD

Ana A Baumann http://orcid.org/0000-0002-4523-0147

\section{REFERENCES}

1 GBD 2017 Risk Factor Collaborators. Global, regional, and national comparative risk assessment of 84 behavioural, environmental and occupational, and metabolic risks or clusters of risks for 195 countries and territories, 1990-2017: a systematic analysis for the global burden of disease study 2017. Lancet 2018;392:1923-94.

2 Roth GA, Mensah GA, Johnson CO, et al. Global burden of cardiovascular diseases and risk factors, 1990-2019: update from the GBD 2019 study. J Am Coll Cardiol 2020;76:2982-3021.

3 Unger T, Borghi C, Charchar F, Thomas U, Claudio B, Fadi C, et al. 2020 International Society of hypertension global hypertension practice guidelines. Hypertension 2020;75:1334-57.

4 WHO. Raised blood pressure. WHO, 2020. Available: https://www. who.int/gho/ncd/risk_factors/blood_pressure_prevalence_text/en/

5 Lloyd-Sherlock P, Beard J, Minicuci N, et al. Hypertension among older adults in low- and middle-income countries: prevalence, awareness and control. Int J Epidemiol 2014:43:116-28.

6 Forouzanfar MH, Liu P, Roth GA, et al. Global burden of hypertension and systolic blood pressure of at least 110 to $115 \mathrm{MM} \mathrm{Hg}, 1990-$ 2015. JAMA 2017;317:165-82.

7 Nahimana M-R, Nyandwi A, Muhimpundu MA, et al. A populationbased national estimate of the prevalence and risk factors associated with hypertension in Rwanda: implications for prevention and control. BMC Public Health 2017;18:2.

8 Rwanda Ministry of Health. Ministry of health, 2020. Available: https://www.moh.gov.rw/

9 Twagirumukiza M, De Bacquer D, Kips JG, et al. Current and projected prevalence of arterial hypertension in sub-Saharan Africa by sex, age and habitat: an estimate from population studies. $J$ Hypertens 2011;29:1243-52.

10 NCD Risk Factor Collaboration (NCD-RisC). Worldwide trends in blood pressure from 1975 to 2015: a pooled analysis of 1479 population-based measurement studies with $19 \cdot 1$ million participants. Lancet 2017;389:37-55.

11 World Health Organization. World Heakth rankings. World life expectancy, 2016. Available: https://www.worldlifeexpectancy.com/ world-health-rankings

12 de Ramirez SS, Enquobahrie DA, Nyadzi G, et al. Prevalence and correlates of hypertension: a cross-sectional study among rural populations in sub-Saharan Africa. J Hum Hypertens 2010;24:786-95.

13 Baumann A, Hooley C, Mutabazi V. Training implementation research in Rwanda: evaluation, challenges and lessons learned. in: AcademyHealth, 2019. Available: https://academyhealth.confex.com/ academyhealth/2019di/meetingapp.cgi/Paper/35206

14 Kirchner JE, Smith JL, Powell BJ, et al. Getting a clinical innovation into practice: an introduction to implementation strategies. Psychiatry Res 2020;283:112467.

15 Powell BJ, Beidas RS, Lewis CC, et al. Methods to improve the selection and tailoring of implementation strategies. J Behav Health Serv Res 2017;44:177-94.

16 Bergström A, Skeen S, Duc DM, et al. Health system context and implementation of evidence-based practices-development and validation of the context assessment for community health (coach) tool for low- and middle-income settings. Implement Sci 2015;10:120.

17 Duc DM, Bergström A, Eriksson L, et al. Response process and testretest reliability of the context assessment for community health tool in Vietnam. Glob Health Action 2016;9:31572.

18 World Health Organization. WHO | process of translation and adaptation of instruments. WHO, 2021. Available: https://www.who. int/substance_abuse/research_tools/translation/en/

19 Niyonsenga SP, Park PH, Ngoga G, et al. Implementation outcomes of national decentralization of integrated outpatient services for 
severe non-communicable diseases to district hospitals in Rwanda. Trop Med Int Health 2021;26:953-61.

20 Tapela NM, Bukhman G, Ngoga G, et al. Treatment of noncommunicable disease in rural resource-constrained settings: a comprehensive, integrated, nurse-led care model at public facilities in Rwanda. Lancet Glob Health 2015;3:S36.
21 Mocumbi S, McKee K, Munguambe K, et al. Ready to deliver maternal and newborn care? Health providers' perceptions of their work context in rural Mozambique. Glob Health Action 2018;11:1532631. 